\title{
Modulation of Metal Species as Early Control Point for Ni-catalyzed Stereodivergent Semihydrogenation of Alkynes with Water
}

\section{Yuanqi Wu}

Changchun University of Technology

\section{Yuhui Ao}

Changchun University of Technology

Chunhui Liu

Xuchang University

Jinbo Zhao

Changchun University of Technology

\section{Wenyu Gao}

Changchun University of Technology

Xuemeng Li

Changchun University of Technology

Hui Wang

Changchun University of Technology

Yongsheng Liu

Changchun University of Technology

Yu Liu ( $\nabla$ yuliu@ccut.edu.cn )

Changchun University of Technology

\section{Article}

Keywords: Olefinic Isomers, Nontoxic Catalysts, Orthogonal Catalytic Pathways, Stereoisomers

Posted Date: May 21st, 2021

DOl: https://doi.org/10.21203/rs.3.rs-464543/v1

License: (c) (i) This work is licensed under a Creative Commons Attribution 4.0 International License. Read Full License

Version of Record: A version of this preprint was published at Nature Communications on March 24th, 2023. See the published version at https://doi.org/10.1038/s41467-023-37022-w. 


\section{Abstract}

A base-assisted metal species modulation mechanism enables Ni-catalyzed stereodivergent semihydrogenation of alkynes with water, delivering both olefinic isomers smoothly using cheap and nontoxic catalysts and additives. Different from most precedents, in which $E$-alkenes derive from the isomerization of $Z$-alkene products, the isomers were formed in orthogonal catalytic pathways. Mechanistic studies suggest base as a key early element in modulation of the reaction pathways: by adding different bases, nickel species with disparate valence states could be accessed to initiate two catalytic cycles toward different stereoisomers. The practicability of the method was showcased with nearly 70 examples, including internal and terminal triple bonds, enynes and diynes, affording semihydrogenated products in high yields and selectivity.

\section{Introduction}

Divergent catalysis as a particularly appealing strategy from both academic and practical perspectives allows convenient control over selectivity towards different terminal products starting from the same material. ${ }^{1-7}$ Predictably, it would be more beneficial for the distinction of reactivity and selectivity if the two target molecules are achieved in separate mechanistic pathways, which generally requires employment of different catalysts to initiate diverse catalytic cycles. Therefore, it would be mechanistically interesting and also operationally practical to develop novel strategies in which different catalytic species could be generated from the same catalyst precursor by simple adjustment of the reaction factors, leading to different products with high selectivity in two independent catalytic cycles.

Transition metal-catalyzed stereodivergent hydrogen transfer of alkynes to produce both Z-and E-olefins have attracted remarkable interests in recent years. ${ }^{8-13}$ Most pioneering examples actualize this transformation by regulation of catalytic systems to realize a $Z$ to $E$ isomerization process at the late stage (Scheme 1, above). For instance, Moran, et al showed that Ni-catalyzed $\mathrm{TH}$ of alkynes with $\mathrm{HCO}_{2} \mathrm{H}$ selectively afforded Z-olefins, which isomerized to $E$-isomers by adding triphos ligand. ${ }^{8}$ Another catalystmodulated system was disclosed by Liu and coworkers in 2016, in which both isomers could be achieved using Co catalysts supported with specified bidentate ligands. The isomerization of Z-alkenes was suppressed by introducing bulky ligand due to the sterically unfavored coordination and insertion processes. ${ }^{9}$ Recently Mei, et al. reported that Pd-catalyzed semihydrogenation of alkynes with $\mathrm{H}_{2} \mathrm{O}$ delivered cis-olefins at room temperature in $\mathrm{CH}_{3} \mathrm{CN}$, while isomerization of the double bond towards transolefins was facilitated at $80^{\circ} \mathrm{C}$ in DMF. ${ }^{10}$ Mechanistically, E-alkenes in the majority of reported strategies originate from the $Z$-isomer, requiring subtleness of the reaction conditions and the steric or electronic properties of the substrates. Therefore, mechanistically orthogonal stereodivergent semi-reduction of alkynes to both olefinic isomers, in which E-alkenes are generated directly from alkynes instead of the isomerization from Z-alkenes, is undoubtedly meaningful in both academic and practical point of views. We envisioned that modulating the catalytic species at an early stage might initiate independent profiles to deliver both isomers in orthogonal manners (Scheme 1, bottom). Ideally, several issues should be 
addressed: (a) independent catalytic cycles should be initiated by simple adjustment of the reaction factor(s) to enable high yield and stereoselectivity; ${ }^{8-10}$ (b) nonprecious metals and ligands without toxic additives would be more favorable; ${ }^{14-18}$ (c) water is the first choice of the hydrogen donor for TH process; ${ }^{10,19-22}$ (d) alkynes with various substituents should be hydrogenated in high yield and stereoselectivity in mild conditions, and over-reduction to saturated alkanes need to be avoided. ${ }^{23,24}$ Based on our interest in Ni-catalyzed reaction and alkyne transformations, ${ }^{25-27}$ We launched a project with nickel catalysts to address the above challenges. After laborious trials, we realized an unprecedented $\mathrm{Ni}$-catalyzed stereodivergent $\mathrm{TH}$ of alkynes with water in a novel controlling mode, in which the key to the success of modulation is the judicious inclusion of the base. Notably, unlike most existing reports, formation of trans-olefins was unrelated to the isomerization of cis-olefin. Mechanistic investigations suggested that base modulated the valence state of active nickel species derived from the same simple pre-catalyst. Consequently, the isomers were achieved independently in completely disparate catalytic pathways: the in situ formed $\mathrm{Ni}(\mathrm{II})$ species delivered Z-alkenes, while the $\mathrm{Ni}(\mathrm{I})$ species selectively afforded E-alkenes as final products.

\section{Results}

Optimization of the reaction conditions. We initiated our exploration by evaluating the transfer hydrogenation of the model substrate 1a with simple nickel sources and 2,2'-bipyridine ligands (Table 1). The first obstacle to overcome is the activation of the inert $\mathrm{H}_{2} \mathrm{O}$ molecule in our nickel catalyst system. ${ }^{28-}$ ${ }^{30}$ Gratifyingly, boron reagents showed unique effect, and the alkenes were obtained in high yield and selectivity using $\mathrm{Na}_{2} \mathrm{CO}_{3}$ as base. $\mathrm{B}_{2}$ pin ${ }_{2}$ turned out to be more efficient than other diboron compounds

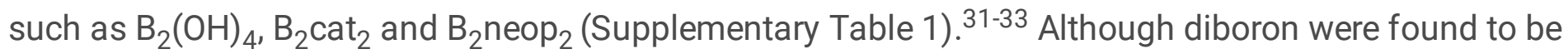
capable of activating water in Pd-catalyzed systems, ${ }^{31-35}$ including hydrogenation of unsaturated $\mathrm{C}-\mathrm{C}$ bonds to saturated alkanes, ${ }^{31}$ it is, as far as we know, the first case for such activation effect in $\mathrm{Ni}$ catalyst systems. Notably, E-alkene 3 a was formed as the major isomer, and over-reduced alkane product was not observed. Solvents turned out to exert a profound influence on the reactivity (Supplementary Table 1), $72 \%$ yield of alkenes were obtained with 11/89 isomeric ratio in DMF (entry 1 ). Decorating the bipyridine ligand with electron-withdrawing ester groups totally suppressed the reactivity (entry 2). Subsequent screening of other bipyridine derivatives as well as phenanthroline ligands L3-L6 provided comparatively inferior results to 2,2'-bipyridine (entries 3-6). Systematic screening of nickel catalyst, ligand, base, boron and water (Supplementary Table 2 and 3) showed that base exerted an unexpected, yet decisive role in the control of selectivity. As shown in Table 1, the reaction was evidently inclined to $E-$ selectivity by $\mathrm{K}_{2} \mathrm{CO}_{3}, \mathrm{NaOH}$ and $\mathrm{CF}_{3} \mathrm{CO}_{2} \mathrm{Na}$, with the later showing the best result, affording $3 \mathrm{a}$ in $84 \%$ isolated yield and 6/94 Z/E ratio (entries 7-9). Interestingly, a slant to Z-selectivity was shown with $\mathrm{CH}_{3} \mathrm{CO}_{2} \mathrm{Na}$, providing $2 \mathrm{a}$ with $69 / 31 \mathrm{Z} / \mathrm{E}$ ratio (entry 10 ). Organic bases such as DABCO and $\mathrm{Et}_{3} \mathrm{~N}$ were also tested, and $E$-alkene 3 a was delivered as the major product (entries 11 and 12). The catalyst loading could be lowered to $5 \mathrm{~mol} \%$ with no erosion of the yield or selectivity (entry 13). The reactivity was almost totally shut down at a lower temperature of $60^{\circ} \mathrm{C}$ (entry 14), which might due to the insufficient energy 
for the isomerization of alkenyl nickel intermediate (Figure 1i). In contrast, comparable results were observed at higher temperatures (entries 15 and16).

The above results inspired us to further proceed with other bases aiming at the optimization for $Z$ selective semihydrogenation of $1 \mathrm{a}$. As shown in Table $2, \mathrm{CH}_{3} \mathrm{CO}_{2} \mathrm{~K}$ and $\mathrm{CH}_{3} \mathrm{CO}_{2} \mathrm{Cs}$ acted similarly as $\mathrm{CH}_{3} \mathrm{CO}_{2} \mathrm{Na}$, indicating that metal ions are not responsible for the selectivity reversal (entries 1 and 2). Only moderate selectivity was achieved when $\mathrm{HCO}_{2} \mathrm{Na}$ was added (entry 3). To our delight, $\mathrm{PhCO}_{2} \mathrm{Na}$ gave a promising result, providing the final olefins in $80 / 20$ selectivity (entry 4). Again, dicarboxylate ligand L2 showed dramatically decreased reactivity (entry5). In contrast, 4,4'-dimethoxy-2,2'-bipyridine L3 improved the selectivity to 93/7 (entry 6). Ligands L4 and L5 bearing methyl groups at 3,3'- or 4,4'positions both gave slightly reduced selectivity than L3 (entries 7 and 8). When the loading of the catalyst and base were reduced, alkenes were retrieved in slightly improved yield and selectivity (entries 9 and 10). Contrary to E-selective system (Table 1 , entry 14 ), the reaction could still proceed smoothly at a lower temperature, albeit 1a was partially recovered (entry 11). Performing the reaction at higher temperatures resulted in poorer selectivities (entries 12 and 13).

Mechanistic investigations. Several questions deserve exploration to better understand this unprecedented system: (a) is water in the system indeed the hydrogen donor? (b) are alkenes generated from hydrometallation of in situ formed $\mathrm{Ni}-\mathrm{H}$ species or hydrolysis of vinyl boron compounds? (c) does isomerization of Z-olefins take effect similarly as most precedents to afford E-olefins? (d) what are the roles of the bases in modulation of the reaction outcomes? To answer these questions, a series of mechanistic studies were carried out. Firstly, deuterium-labeled experiments were conducted (Figure 1a). The deuterium was incorporated into both the 1,2-olefinic positions of $\mathbf{2} \mathbf{a}^{\mathbf{\prime}}$ and $\mathbf{3} \mathbf{a}$ ' with $\mathrm{D}_{2} \mathrm{O}$ instead of $\mathrm{H}_{2} \mathrm{O}$ under both standard conditions (equations (1) and (2)). Similar results were also observed for unsymmetric alkynes $\mathbf{1 z}$ and $\mathbf{1} \mathbf{g}$, with the former leading to even higher deuterations (equations (7) and (8)). In contrast, there was no sign of deuteration on the products using DMF- $d_{7}$ as solvent (equations (3) and (4)). When the reactions of $1 \mathrm{a}$ using $\mathrm{D}_{2} \mathrm{O}$ were placed in hydrogen atmosphere, comparative deuterium isotopic contents as in argon were observed (equations (5) and (6)), proving that releasing of $\mathrm{H}_{2}$ and consequent hydrogenation was not involved in the catalytic pathway. Control experimental studies of vinylboron reagents $\mathbf{4}$ and $\mathbf{5}$ were performed under the standard reaction conditions. ${ }^{36,37}$ Olefin products $\mathbf{2} \mathbf{a}$ and $\mathbf{3} \mathbf{a}$ were not detected, excluding the possibility of hydrolysis of vinylboron derivatives (Figure $1 \mathrm{~b}$, equations (9) and (10)). This, together with the reactions under $\mathrm{H}_{2}$ atmosphere, indicated that $\mathrm{Ni}-\mathrm{H}$ species were formed between the nickel pre-catalyst and $\mathrm{H}_{2} \mathrm{O}$ assisted by $\mathrm{B}_{2} \mathrm{pin}_{2}$, which would deliver alkenyl nickel intermediates to accomplish the catalytic cycle.

To deeper understand the process of selective semi-reduction, the kinetic behavior of the reaction system was monitored (Fig.1c). The kinetic profile of Z-selective semihydrogenation showed that $2 \mathrm{a}$ was generated by degrees in the initial 5 hours, and the yield stayed closely aligned with the conversion. After this period, 3 a began to show up and gradually increased to $6 \%$ yield, alongside with a sharp decline of the yield growth rate of $\mathbf{2 a}$ (Fig.1c, left). We postulate that the small amount of $E$-alkene in this system 
derives from isomerization of the Z-isomer, which was suppressed in the initial 5 hours since competitive coordination of alkyne 1a with the metal center. Consumption of most 1a after 5 hours left space for the coordination of $\mathbf{2} \mathbf{a}$ for the subsequent isomerization process, which still need $\mathbf{1} \mathbf{a}$ as auxiliary since the selectivity remained unchanged after disappearance of $1 \mathrm{a}$. The $E$-selective reaction profile with $\mathrm{CF}_{3} \mathrm{CO}_{2} \mathrm{Na}$ as base clearly indicated the nonexistence of $Z / E$ isomerization (Fig.1c, right). Approximately $6 \%$ of $Z$-alkene was already formed at the early stage of the reaction, which maintained in this level until 1a was completely converted. The concentration of $\mathbf{3 a}$ increased gradually, which was independent with the amount of $2 \mathbf{a}$.

To further verify the above inferences, a series of control experiments were conducted (Fig.1d). When Zalkene 2 a was put in both standard conditions, only less than $5 \%$ of $E$-alkene was detected (equations (11) and (12)), demonstrating the reluctance of the $Z / E$ isomerization in these conditions. Elevating the reaction temperature showed a beneficial effect for the isomerization, which was promoted to $13 \%$ by heating $2 \mathbf{a}$ at $120^{\circ} \mathrm{C}$ under the $Z$-selective condition (equation (13)). Consistently, the reaction of $1 \mathbf{a}$ at $120^{\circ} \mathrm{C}$ under this condition afforded the corresponding olefinic products in 86/14 selectivity (equation (14)), compared with $93 / 7$ at $80^{\circ} \mathrm{C}$.

The color changes between the two reaction systems were significantly different. As shown in Figure 1e, the Z-selective system seemed turbid and beige at the very beginning, which turned to light brown after several minutes and got darker later. The color changed to tan-yellow gradually in about one hour and became lighter to milk-white after another one hour, which remained till the end. A completely different visual appearance mutation was observed for the $E$-selective system, which looked transparent black and got darker quickly at the very early stage. Interestingly, as soon as the reaction was over as monitored of the crude mixture, the color changed to bright yellow immediately, which could be regarded as a simple hint for the complete of the reaction. We postulate that the dark color ascribes to the coordination of the triple bond to the metal center, which was terminated promptly once alkynes were exhausted. ${ }^{24}$ The distinction in colors of the two systems indicates that different nickel species might be involved, leading to the corresponding olefinic products in totally unrelated pathways. The color variation of the control experiments on base was quite similar to the above observation (Fig. 1e, bottom): the initial pale green color changed to tint of turbidity yellow and clarify black color separately after addition of $\mathrm{PhCO}_{2} \mathrm{Na}$ and $\mathrm{CF}_{3} \mathrm{CO}_{2} \mathrm{Na}$, respectively, indicating the formation of different nickel species was modulated with the choice of base.

Competitive control experiments of the bases were conducted to further illustrate their functions (Figure 1f). After the standard $Z$-selective mixture using $\mathrm{PhCO}_{2} \mathrm{Na}$ was stirred for $1 \mathrm{~h}$, another 2.0 equivalent of $\mathrm{CF}_{3} \mathrm{CO}_{2} \mathrm{Na}$ was added, and no apparent influence on the reaction outcomes was observed (equation (15)). By contrary, a worse selectivity was caused by addition of $\mathrm{PhCO}_{2} \mathrm{Na}$ into the E-selective system (28/72 vs $4 / 96)$ (equation (16)). 
All the mechanistic insights and the visual phenomenon pointed to distinct catalytic pathways for the two reaction systems, inspiring us to further inquire whether different metal species were taking effect inherently. To detect whether nanoparticles were involved in our Ni-B- $\mathrm{H}_{2} \mathrm{O}$ system, general mercury drop experiments were performed ${ }^{32,34}$ (Figure $1 \mathrm{~g}$ ). The yield or selectivity was not affected in either systems (equations (17) and (18)), excluding heterogeneous catalytic pathways. Despite the failure in capture of metallic intermediates, electron paramagnetic resonance (EPR) analyses provided clues on the active nickel species and the base effect. As shown in Figure 1h (2), strong EPR signals were observed in the $E$ selective mixture, indicating the formation of $\mathrm{Ni}(\mathrm{I})$ or $\mathrm{Ni}(\mathrm{III})$ species. ${ }^{38-41}$ The signals of such $\mathrm{Ni}$ species could not be found at ambient temperature, which is in accordance with our experimental observations that semihydrogenations of 1a were not permitted at it (Supplementary Table 3, entry 25). In contrast, EPR active species was not observed in Z-selective system (Figure $1 \mathrm{~h}(1)$ ), featuring a $\mathrm{Ni}(0) / \mathrm{Ni}(\mathrm{II})$ catalytic cycle. In agreement with the competitive experiments of bases (equation (16)), the EPR signals for the reactions using $\mathrm{CF}_{3} \mathrm{CO}_{2} \mathrm{Na}$ as base were markedly weakened after the addition of $\mathrm{PhCO}_{2} \mathrm{Na}$ (Figure $1 \mathrm{~h}(3)$ ). In line with the fact that use of $\mathrm{HCO}_{2} \mathrm{Na}$ as base gave an almost 1:1 ratio of the $Z$ - and $E$ alkenes (Table 2, entry 3), the EPR signal of the system with $\mathrm{HCO}_{2} \mathrm{Na}$ was less significant than that with $\mathrm{CF}_{3} \mathrm{CO}_{2} \mathrm{Na}$ (Figure $1 \mathrm{~h}(4)$ ), but much more significant than that of $\mathrm{PhCO}_{2} \mathrm{Na}$ system (Figure $1 \mathrm{~h}(1)$ ).

Although more experimental supports are awaited to uncover the detailed mechanism, a general scenario could be delineated based on the above results and relative literatures ${ }^{31,32,42-50}$ (Figure 1i): $\mathrm{NiBr}_{2}$ would interact with the bases firstly, delivering carboxylates carrying different counter anions. The difference in electronic properties between the benzoate and the trifluoroacetate endows them with distinct reactivities towards $\mathrm{B}_{2} \mathrm{pin}_{2}$. Consequently, $\mathrm{Ni}(\mathrm{II})$ species $\mathbf{C}$ is generated directly from the benzoate $\mathbf{B}$ and $\mathrm{B}_{2} \mathrm{pin}_{2}$. Activation of $\mathrm{H}_{2} \mathrm{O}$ molecule delivers $\mathrm{Ni}(\mathrm{II})$ - $\mathrm{H}$ species $\mathrm{D}$, which undergo syn-addition to the triple bond to afford alkenyl $\mathrm{Ni}$ (II) intermediate $\mathrm{E}$. Participation of another $\mathrm{H}_{2} \mathrm{O}$ molecule release the cis-olefin and regenerate $\mathbf{C}$ with the assistance of $\mathrm{B}_{2} \mathrm{pin}_{2}$. Based on the kinetic experiments, coordination and insertion of the Z-alkene to the $\mathrm{Ni}$-H species assisted by alkyne precursor would occur in the late stage of the reaction, followed by isomerization process resulting in slight stereo-impurity. We propose that isomerization of a vinyl $\mathrm{Ni}(\mathrm{I})$ species is responsible for the E-selectivity observed in this approach, the specific oxidation state at Ni could provide an opportunity for isomerization ${ }^{42,43}$. At the beginning of the cycle, $\mathrm{Ni}(0)$ species $\mathbf{H}$ might be generated firstly from nickel trifluoroacetate $\mathbf{G}$ and $\mathrm{B}_{2} \mathrm{pin}_{2}$. Oxidative addition of another molecule of $\mathrm{B}_{2}$ pin $_{2}$ furnishes $\mathrm{Ni}$ (II) species $\mathbf{C}$, comproportionation between $\mathbf{C}$ and $\mathbf{H}$ occurs instantly, forging $\mathrm{Ni}(\mathrm{I})$ species I to initiate the catalytic cycle. Activation of $\mathrm{H}_{2} \mathrm{O}$ molecule would deliver $\mathrm{Ni}(\mathrm{I})-\mathrm{H}$ species $\mathbf{J}$, followed by insertion of alkyne leading to vinyl $\mathrm{Ni}(\mathrm{I})$ intermediate $\mathbf{K}$, which may undergo isomerization ${ }^{43}$ to $E$-alkenyl nickel intermediate $\mathbf{L}$. Thermodynamically more stable product $\mathbf{3}$ is generated by hydrolysis of $\mathbf{L}$, and the acquired nickel hydroxide $\mathbf{M}$ could be transformed back to $\mathrm{Ni}(\mathrm{I})$ species I in the aid of $\mathrm{B}_{2} \mathrm{pin}_{2}$.

Substrate scope. The synthetic practicability of this system was sufficiently embodied in the functional group compatibility investigations. In Table 3a, the Z-selective semi-reduction of various alkynes 1 using 
$\mathrm{PhCO}_{2} \mathrm{Na}$ as base is summarized. This reaction proceeded successfully toward substituted diarylethynes bearing a diverse set of substituents. Specifically, substrates bearing methyl or tert-butyl groups at $p$ - or $m$-positions all worked smoothly under the standard conditions (2a-2d), as well as hindered isopropyl (2e) or phenyl (2f) groups located in the ortho-position of the aryl terminus, suggesting the insensitivity of the system to steric effect. Electron-donating methoxy substituent was well accommodated, and the diaryl alkenes were generated in high yields and selectivity (2h, $\mathbf{2} \mathbf{i}$ and $\mathbf{2} \mathbf{j})$. Amino functional group $\mathbf{2 k}$ was no exception, well tolerated in this catalytic semihydrogenation process. Z-olefins with electron-withdrawing trifluoromethyl (1), cyano (1m, 1n), ester (10) and acyl (1p) groups could also be achieved uneventfully. Fluoro- and chloro-containing products (2q-2t) were furnished from the corresponding alkynes, leaving space for further functionalization. The generality of the system was further showcased by the tolerance of naphthyl (2u) and heterocycles including thienyl (2v), benzofuryl $(2 \mathbf{w})$ and pyridyl motifs $(2 \mathbf{x})$, particularly the latter, considering pyridinyl ligands were used in our catalytic system. Moreover, running in a longer reaction time or higher temperature, alkynes carrying both naphthenic and linear alkyl terminuses could be reduced to the corresponding olefinic products efficiently (2z-2ff). Notably, only Z-alkenes were formed specifically from the alkyl substrates, supporting our previous deduction that the E-alkenes in the $Z$-selective conditions might derive from the isomerization process, which was sluggish for alkyl alkenes due to their weak coordinating ability to the metallic species. The compatibility of the system was further underlined by successful involvement of unprotected primary $\mathrm{OH}$ group (2dd), which was unaffected under the catalytic conditions. Natural product derived alkyne with estrone skeleton proceeded smoothly, and the desired product 2ee was furnished in excellent yield and selectivity. Finally, internal alkyne 1ff bearing 1,2-dialkyl substituents also gave high yield and perfect stereoselectivity.

A survey on the substrate scope was performed next to demonstrate the robustness of the $E$-selective $\mathrm{TH}$ process using $\mathrm{CF}_{3} \mathrm{CO}_{2} \mathrm{Na}$ as base (Table $3 \mathrm{~b}$ ). Similar as the former system, diaromatic internal alkynes with a wide range of functional groups such as methyl (1a-1c), tert-butyl (1d), isopropyl (1e), methoxyl (1i, 1j), amino (1k), trifluoromethyl (1l), cyano (1m), ester (10), acyl (1p) and halogen substituents (1q-1t) were all hydrogenated to the desired trans-alkenes uneventfully. Heteroaromatic rings including thienyl (1v), benzofuryl (1w) and pyridyl (1x, 1y) substituents were compatible again, delivering the alkenyl heterocycles selectively. The reaction of alkyl acetylene was more challenging, affording alkene $\mathbf{3 g g}$ in moderate yield and inferior selectivity. Propargylic esters were transformed to E-olefins (3hh-3jj) as single isomers in moderate yields and excellent selectivity. Consistent with the previous observation, for all the E-selective experiments, a mutation of color from black to bright yellow was observed as soon as the reaction finished.

Finally, we tested the reactivity of terminal alkynes, which are more inclined to over-reduction. As shown in Table 3c, alkene 6 was obtained in high yield in Z-selective conditions from $1 \mathbf{k k}$, and saturated ethyl product was not observed. The condition could also be extended to diynes $1 \mathrm{ll}$ and $1 \mathrm{~mm}$, with both triple bonds being hydrogenated in high selectivity. Interestingly, the reaction of conjugated enyne $1 \mathrm{nn}$ in $Z$ selective conditions afforded diene 9 with E-configuration as the major product. On the contrary, Z-enyne 10 was obtained in high selectivity when 1,3-diyne 100 was loaded in E-selective conditions. 


\section{Conclusions}

In conclusion, we have disclosed an unprecedented Ni-catalyzed stereodivergent semihydrogenation of acetylenes with water. The configuration of the olefinic products was controlled by the choice of bases, which were demonstrated to influence the valence states of the catalytic nickel species. Consequently, $E$ alkenes were achieved independently from the direct reduction of alkyne precursors instead of isomerization of the $Z$-isomers. The strategy also features use of cheap catalysts and nontoxic reagents, and the compatibility with an assortment of alkynyl substrates such as internal and terminal alkynes, 1,3enynes and diynes. Besides its significance in semihydrogenation of alkynes, we believe that the mechanistic insights would lead to better understanding of the performance of nickel species, and also pave the way to further exploration of the other transition metal catalyst systems.

\section{Declarations}

\section{Competing interests}

The authors declare no competing interests.

\section{References}

1. Nájera, C., Beletskaya, I. P., \& Yus, M. Metal-catalyzed Regiodivergent Organic Reactions. Soc. Rev. 48, 4515-4618 (2019).

2. Irina P. Beletskaya, I. P., Nájera, C. \& Yus, M. Chemodivergent Reactions. Soc. Rev. 49, 7101-7166 (2020).

3. Ping, L., Chung, D. S., Bouffard, J. \& Lee, S. Transition Metal-catalyzed Site- and Regio-divergent C-H Bond Functionalization. Soc. Rev. 46, 4299-4328 (2017).

4. Mei, L.,Wei, Y.,Tang, X. \& Shi, M. Catalyst-Dependent Stereodivergent and Regioselective Synthesis of Indole-Fused Heterocycles through Formal Cycloadditions of Indolyl-Allenes. Am. Chem. Soc. 137, 8131-8137 (2015).

5. Xu, S. et al. Enantioselective Regiodivergent Synthesis of Chiral Pyrrolidines with Two Quaternary Stereocenters via Ligand-Controlled Copper(I)-Catalyzed Asymmetric 1,3-Dipolar Cycloadditions. Am. Chem. Soc. 140, 2272-2283 (2018).

6. Yuen, O. Y. \& So, C. M. Ligand Control of Palladium-Catalyzed Site-Selective a- and g-Arylation of a,bUnsaturated Ketones with (Hetero)aryl Halides. Chem. Int. Ed. 59, 23438-23444 (2020).

7. Wang, J., Wu, P., Wu, J., Mei, G. \& Shi, F. Chemodivergent Tandem Cyclizations of 2-Indolylmethanols with Tryptophols: C-N versus C-C Bond Formation. Org. Chem. 83, 5931-5946 (2018).

8. Richmond, E. \& Moran, J. Ligand Control of E/Z Selectivity in Nickel-Catalyzed Transfer Hydrogenative Alkyne Semireduction. Org. Chem. 80, 6922-6929 (2015).

9. Fu, S. et al. Ligand-Controlled Cobalt-Catalyzed Transfer Hydrogenation of Alkynes: Stereodivergent Synthesis of Z- and E-Alkenes. Am. Chem. Soc. 138, 8588-8594 (2016). 
10. Zhao, C. Q. et al. Water as a Hydrogenating Agent: Stereodivergent Pd-Catalyzed Semihydrogenation of Alkynes. Lett. 21, 1412-1416 (2019).

11. Li, J. \& Hua, R. Stereodivergent Ruthenium-Catalyzed Transfer Semihydrogenation of Diaryl Alkynes. Eur. J. 17, 8462-8465 (2011).

12. Chen, T., Xiao, J., Zhou, Y., Yin, S. \& Han, L. B. Nickel-catalyzed (E)-selective Semihydrogenation of Internal Alkynes with Hypophosphorous Acid. Organomet. Chem. 749, 51-54 (2014).

13. Li, K. et al. Cobalt Catalyzed Stereodivergent Semi-hydrogenation of Alkynes using $\mathrm{H}_{2} \mathrm{O}$ as the Hydrogen Source, Commun. 55, 5663-5666 (2019).

14. Srimani, D. et al. Iron Pincer Complex Catalyzed, Environmentally Benign, E-Selective SemiHydrogenation of Alkynes. Chem. Int. Ed. 52, 14131-14134 (2013).

15. Cortese, N. A. \& Heck, R. F. Palladium-catalyzed Reductions of $a, b$-unsaturated Carbonyl Compounds, Conjugated Denes, and Acetylenes with Trialkylammonium Formats. Org. Chem. 43, 3985-3987 (1978).

16. Semba, K., Fujihara, T., Xu, T. H., Terao, J. \& Tsuji, Y. Copper-Catalyzed Highly Selective Semihydrogenation of Non-Polar Carbon-Carbon Multiple Bonds Using a Silane and an Alcohol. Synth. Catal. 354, 1542-1550 (2012).

17. Whittaker, A. M. \& Lalic, G. Monophasic Catalytic System for the Selective Semireduction of Alkynes. Lett. 15, 1112-1115 (2013).

18. Wang, D. \& Astruc, D. The Golden Age of Transfer Hydrogenation. Rev. 115, 6621-6686 (2015).

19. Richards, E. M. \& Tebby, J. C. Reactions of phosphines with acetylenes. Part VIII. Synthesis of 1,2dideuteriated olefins. Chem. Soc. (C) 1542-1544 (1969).

20. Chou, W., Clark, D. L. \& White, J. B. The Use of Rieke Zinc Metal in the Selective Reduction of Alkynes. Tetrahedron Lett. 32, 299-302 (1991).

21. Kataoka, Y., Takai, K., Oshima, K. \& Utimoto, K. Selective Reduction of Alkynes to (Z)-alkenes via Niobium- or Tantalum-alkyne Complexes. Org. Chem. 57, 1615-1618 (1992).

22. Chen, Z., Luo, M., Wen, Y., Luo, G. \& Liu, L. Transition-Metal-Free Semihydrogenation of Diarylalkynes: Highly Stereoselective Synthesis of trans-Alkenes Using $\mathrm{Na}_{2} \mathrm{~S} \cdot 9 \mathrm{H}_{2}$ Org. Lett. 16, 3020-3023 (2014).

23. van Laren, M. W. \& Elsevier, C. J. Selective homogeneous palladium(0)-catalyzed hydrogenation of alkynes to (Z)-alkenes. Chem. Int. Ed. 38, 3715-3717 (1999).

24. Wang, Y., Huang, Z. \& Huang, Z. Catalyst as Colour Indicator for Endpoint Detection to Enable Selective Alkyne trans-hydrogenation with Ethanol, Nat. Catal. 2, 529-536 (2019).

25. Gao, J. et al. Catalytic C-C Cleavage/Alkyne-Carbonyl Metathesis Sequence of Cyclobutanones. Lett. 22, 3993-3999 (2020).

26. Liu, Y., Cornella, J. \& Martin, R. Ni-catalyzed Carboxylation of Unactivated Primary Alkyl Bromides and Sulfonates with $\mathrm{CO}_{2}$. Am. Chem. Soc. 136, 11212-11215 (2014). 
27. Wang, X., Liu, Y. \& Martin, Ruben. Ni-Catalyzed Divergent Cyclization/Carboxylation of Unactivated Primary and Secondary Alkyl Halides with $\mathrm{CO}_{2}$. Am. Chem. Soc. 137, 6476-6479 (2015).

28. Sato, T., Shoji Watanabe, S., Kiuchi, H., Oi, S. \& Yoshio Inoue, Y. Hydrogenation of Olefins Using Water and Zinc Metal Catalyzed by a Rhodium Complex. Tetrahedron Lett. 47, 7703-7705 (2006).

29. Muhammad, O., Sonavane, S. U., Sasson, Y. \& Chidambaram, M. Palladium/Carbon Catalyzed Hydrogen Transfer Reactions using Magnesium/Water as Hydrogen Donor. Lett. 125, 46-51 (2008).

30. Yan, M. et al. Nanoporous Gold Catalyst for Highly Selective Semihydrogenation of Alkynes: Remarkable Effect of Amine Additives. Am. Chem. Soc. 134, 17536-17542 (2012).

31. Cummings, S. P., Le, T. N., Fernandez, G. E., Quiambao, L. G. \& Stokes, B. J. TetrahydroxydiboronMediated Palladium-catalyzed Transfer Hydrogenation and Deuteriation of Alkenes and Alkynes Using Water as the Stoichiometric H or D Atom Donor. Am. Chem. Soc. 138, 6107-6110 (2016).

32. Xuan, Q. \& Song, Q. Diboron-Assisted Palladium-Catalyzed Transfer Hydrogenation of $\mathrm{N}$-Heteroaromatics with Water as Hydrogen Donor and Solvent. Lett. 18, 4250-4253 (2016).

33. Kong, W., Wang, Q. \& Zhu, J. Water as a Hydride Source in Palladium-Catalyzed Enantioselective Reductive Heck Reactions. Chem. Int. Ed. 56, 3987-3991 (2017).

34. Ojha, D. P., Gadde, K. \& Prabhu, K. R. Generation of Hydrogen from Water: A Pd-Catalyzed Reduction of Water Using Diboron Reagent at Ambient Conditions. Lett. 18, 5062-5065 (2016).

35. Flinker, M. et al. Efficient Water Reduction with $\mathrm{sp}^{3}-\mathrm{sp}^{3}$ Diboron(4) Compounds: Application to Hydrogenations, H-D Exchange Reactions, and Carbonyl Reductions. Chem. Int. Ed. 56, 1591015915 (2017).

36. Bao, H., Zhou, B., Jin, H. \& Liu, Y. Diboron-Assisted Copper-Catalyzed Z-Selective Semihydrogenation of Alkynes Using Ethanol as a Hydrogen Donor. Org. Chem. 84, 3579-3589 (2019).

37. Han, X., Hu, J., Chen, C., Yuanb, Y. \& Shi, Z. Copper-catalysed, Diboron-mediated cis-dideuterated Semihydrogenation of Alkynes with Heavy Water. Commun. 55, 6922-6925 (2019).

38. James, T. L., Smith, D. M. \& Holm, R. H. Stereoelectronic Preferences in Electron Transfer Series of Nickel with Tridentate Ligands Containing Hard-Soft Donor Sets. Chem. 33, 4869-4877 (1994).

39. Kim, J. S., Reibenspies, J. H. \& Darensbourg, M. Y. Characteristics of Nickel(0), Nickel(I), and Nickel(II) in Phosphino Thioether Complexes: Molecular Structure and $S$-Dealkylation of $\left(\mathrm{Ph}_{2} \mathrm{P}(0-\right.$ $\left.\left.\mathrm{C}_{6} \mathrm{H}_{4}\right) \mathrm{SCH}_{3}\right)_{2} \mathrm{Ni}^{0}$. Am. Chem. Soc. 118, 4115-4123 (1996).

40. Silver, S. C. et al. Protein Delivery of a Ni Catalyst to Photosystem I for Light-Driven Hydrogen Production. J. Am. Chem. Soc. 135, 13246-13249 (2013).

41. Zhang, C. P. et al. A Five-Coordinate Nickel(II) Fluoroalkyl Complex as a Precursor to a Spectroscopically Detectable Ni(III) Species. J. Am. Chem. Soc. 135, 8141-8144 (2013).

42. Krasovskiy, A. \& Lipshutz, B. H. Ligand Effects on Negishi Couplings of Alkenyl Halides. Lett. 13, 3818-3821 (2011). 
43. Barber, E. R. et al. Nickel-Catalyzed Hydroarylation of Alkynes under Reductive Conditions with Aryl Bromides and Water. Org. Chem. 84, 11612-11622 (2019).

44. Hashimoto, T., Shiota, K. \& Yamaguchi, Y. Selective Synthesis of Secondary Alkylboronates: Markovnikov Selective Hydroboration of Vinylarenes with Bis(pinacolato)diboron Catalyzed by a Nickel Pincer Complex. Lett. 22, 4033-4037 (2020).

45. King, A. E. et al. GordonNi(bpy)(cod): A Convenient Entryway into the Efficient Hydroboration of Ketones, Aldehydes, and Imines. J. Inorg. Chem. 1635-1640 (2016).

46. Kumar, G. R., Kumar, R., Manda R. M. \& Reddy, M. S. A Nickel-catalyzed Anti-carbometallative Cyclization of Alkyne-azides with Organoboronic Acids: Synthesis of 2,3-diarylquinolines. Chem. Commun. 54, 759-762 (2018).

47. Eberhardt, N. A. \& Guan, Nickel Hydride Complexes. Chem. Rev. 116, 8373-8426 (2016).

48. Wang, W., Chao Ding, C. \& Yin, G. Catalyst-controlled Enantioselective 1,1-arylboration of Unactivated Olefins. Catal. 3, 951-958 (2020).

49. Nattmann, L., Saeb, R., Nöthling, N. \& Cornella, J. An Air-stable Binary Ni(0)-olefin Catalyst. Nat. Catal. 3, 6-13 (2020).

50. Clarke, C., Incerti-Pradillos, C. A. \& Lam, H. W. Enantioselective Nickel-Catalyzed anti-Carbometallative Cyclizations of Alkynyl Electrophiles Enabled by Reversible Alkenylnickel E/Z Isomerization. J. Am. Chem. Soc. 138, 8068-8071 (2016).

\section{Figures}




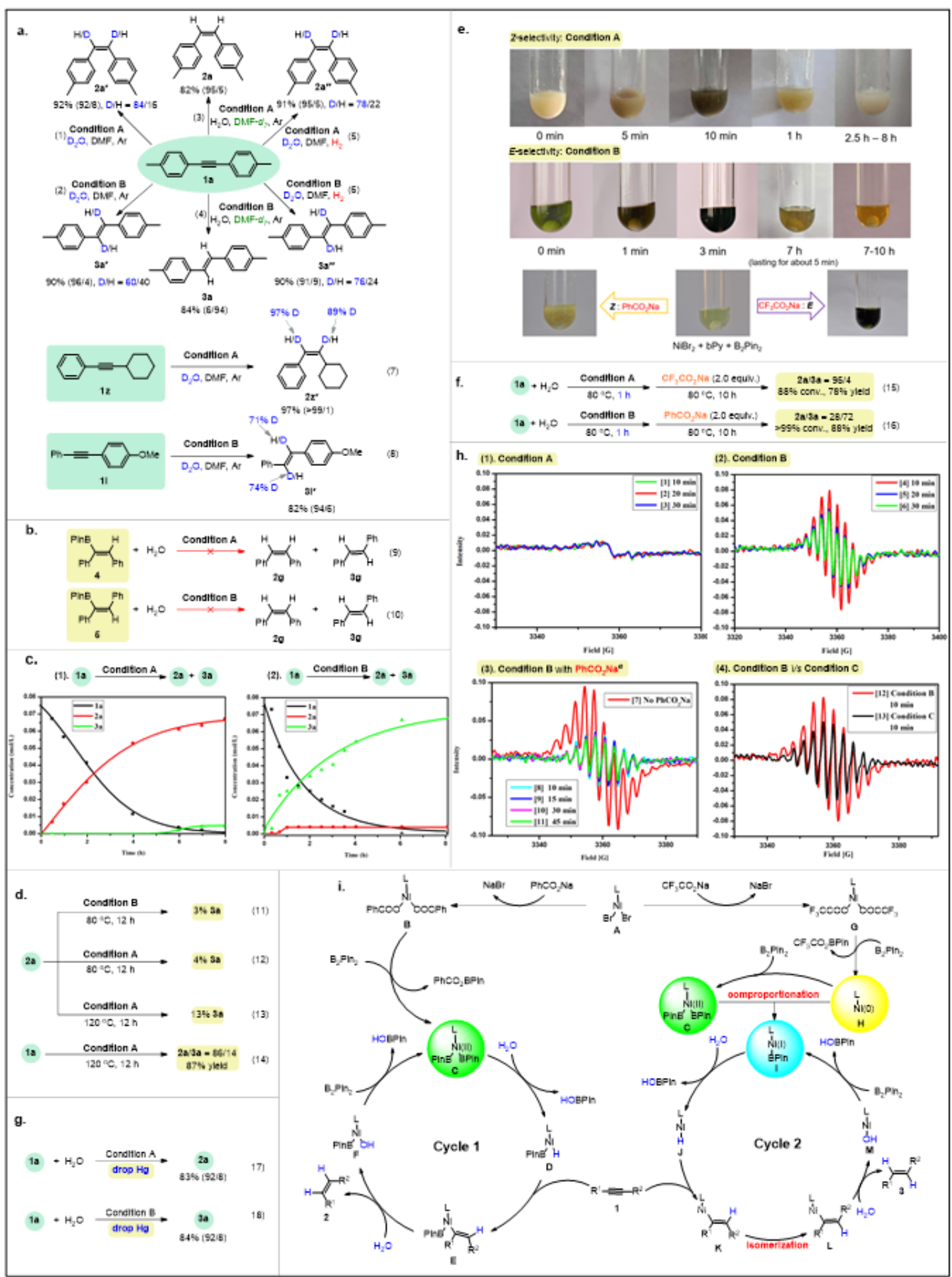

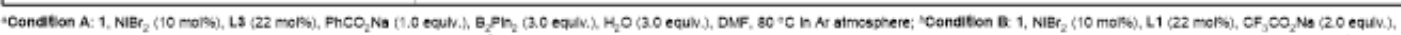

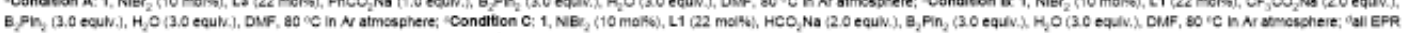

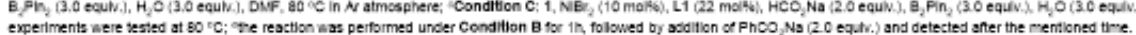

\section{Figure 1}

Mechanistic investigations.

\section{Supplementary Files}

This is a list of supplementary files associated with this preprint. Click to download. 
- supplementarydata.docx

- Scheme1.png

- Table1.png

- Table2.png

- Table3.png

- GraphicalAbstract.png 